\title{
Limitação de atividades, consciência de risco e participação social de pessoas acometidas pela hanseníase
}

\author{
Limitation of activity, risk awareness and social participation of people affected by leprosy \\ Limitación de actividades, conciencia de riesgo y participación social de personas \\ acometidas por la lepra
}

Adriano Rocha Alencar ${ }^{1 *}$, João Maria Corrêa Filho¹, Joana Elisabeth de Sousa Martins Freitas ${ }^{1}$, Lucas Lins Marques².

\section{RESUMO}

Objetivo: Avaliar a limitação de atividade, consciência de risco e participação social de pessoas acometidas pela hanseníase. Métodos: Estudo transversal com amostra de 152 participantes distribuídos em dois grupos. A coleta de dados ocorreu entre novembro de 2018 e março de 2019, mediante aplicação de questionário socioeconômico e dos instrumentos Screening of Activity Limitation and Safety Awareness (SALSA) e Participation Scale. Resultados: Não houve diferença significativa de idade entre os grupos, porém ocorreu associação do sexo masculino e maior frequência de analfabetos entre os multibacilares. Não foi observada associação entre classificação operacional e variáveis socioeconômicas. Quanto aos graus de limitação de atividade, consciência de risco e participação social, observou-se uma diferença maior das médias no grupo dos multibacilares, assim como uma associação entre esse grupo e a presença de consciência de risco envolvido em certas atividades diárias e algum grau de restrição à participação social. Conclusão: Mesmo após o término do tratamento, observaram-se níveis expressivos de limitação de atividade e de grau de consciência de risco, especialmente nos multibacilares. Por fim, a associação das formas multibacilares com a restrição à participação social evidencia como a hanseníase afeta socialmente a vida das pessoas acometidas.

Palavras-chave: Hanseníase, Atividades cotidianas, Participação social, Classificação internacional de funcionalidade, Incapacidade e saúde.

\begin{abstract}
Objective: To evaluate the limitation of activity, risk awareness and social participation of people affected by leprosy. Methods: Cross-sectional study with a sample of 152 participants divided into two groups. Data collection took place between November 2018 and March 2019, through the application of a socioeconomic questionnaire and the instruments Screening of Activity Limitation and Safety Awareness (SALSA) and Participation Scale. Results: It was no significant difference in age between the groups, but there was association of males and a higher frequency of illiterates among multibacillaries. There was no statistically significant association between operational classification and others socioeconomic variables. As for the degrees of activity limitation, risk awareness and social participation, there was a greater difference in the averages in the multibacillary group, as well as a association between this group and the presence of risk awareness involved in certain daily activities and some degree of restriction to social participation. Conclusion: Even after the end of the treatment, significant levels of activity limitation and level of risk awareness were observed, especially in multibacillary patients. Finally, the association of multibacillary forms with the restriction of social participation shows how leprosy socially affects the lives of people affected.
\end{abstract}

Keywords: Leprosy, Daily activities, Social participation, International classification of functionality, Disability and health.

1 Universidade Federal do Piauí (UFPI), Teresina - PI. *E-mail: adrianoralencar@gmail.com

${ }^{2}$ Centro Universitário UniFacid / Wyden, Teresina - PI.

SUBMETIDO EM: 6/2020 | ACEITO EM: 7/2020 ～PUBLICADO EM: 10/2020 


\section{RESUMEN}

Objetivo: evaluar la limitación de la actividad, la conciencia del riesgo y la participación social de las personas afectadas por la lepra. Métodos: estudio transversal con una muestra de 152 participantes divididos en dos grupos. La recopilación de datos tuvo lugar entre noviembre de 2018 y marzo de 2019, utilizando un cuestionario socioeconómico y los instrumentos de detección de limitación de actividad y conciencia de seguridad (SALSA) y escala de participación. Resultados: No hubo diferencias significativas en la edad entre los grupos, pero hubo asociación de hombres y una mayor frecuencia de analfabetos entre los multibacilares. No hubo asociación estadísticamente significativa entre la clasificación operativa y las otros variables socioeconómicas. En cuanto a los grados de limitación de la actividad, la conciencia del riesgo y la participación social, hubo una diferencia mayor en los promedios en el grupo multibacilar, así como una asociación entre este grupo y la presencia de la conciencia del riesgo involucrada en ciertas actividades diarias y cierto grado de restricción a la participación social. Conclusión: Incluso después del final del tratamiento, se observaron niveles significativos de limitación de actividad y nivel de conciencia de riesgo, especialmente en pacientes multibacilares. Finalmente, la asociación de formas multibacilares con la restricción a la participación social muestra cómo la lepra afecta socialmente la vida de las personas afectadas.

Palabras clave: Lepra, Actividades diarias, Participación social, Clasificación internacional de funcionalidad, Discapacidad y salud.

\section{INTRODUÇÃO}

Hanseníase compreende uma doença infectocontagiosa transmitida pelo agente Mycobacterium leprae, que tem predileção por infectar nervos periféricos. Quando não tratada na forma inicial, evolui de forma lenta e progressiva, levando a deformidades e incapacidades físicas (EICHELMANN K, et al., 2013).

Como resultado desse processo, o indivíduo acometido passa a apresentar deficiências, dificuldades na execução de atividades e problemas em situações da vida cotidiana. As incapacidades físicas dificultam a rotina diária em casa e no trabalho, afetando de diversas formas a qualidade de vida e participação social das pessoas acometidas (SANTOS VS, et al., 2015).

Ademais, a limitação de atividades acarreta problemas psicossociais, devido à diminuição da autoestima e do status na comunidade, somados ao estigma da doença, à discriminação e à exclusão social. Essas razões levam à ocultação da condição física, privação da vida social, das atividades de geração de renda e das responsabilidades familiares (SILVA AC, et al., 2014).

Estudos tratam a questão da limitação funcional das pessoas atingidas pela hanseníase, porém, abordam pouco sobre o medo quanto às deficiências ou estigmas associados à doença (ZAMPARONI V, 2017; BRAKEL WH et al., 2006).

Para fins operacionais de tratamento, o Brasil utiliza a classificação adotada pela Organização Mundial de Saúde (OMS), dividindo os casos em paucibacilares e multibacilares. Apresentações clínicas multibacilares são fatores de risco para o desenvolvimento de limitações de atividade funcional, além da presença de reações hansênicas, atraso no diagnóstico e consequente início tardio do tratamento.

Atualmente, um dos desafios críticos no controle da hanseníase são a alta proporção de casos multibacilares e a presença de incapacidades no momento do diagnóstico, indicando atraso na detecção dos casos (GASCHIGNARD J, et al., 2016; MONTEIRO LD, et al., 2014).

Frente às considerações apresentadas, o presente estudo propõe-se a explorar a limitação de atividade, consciência de risco e participação social de pessoas acometidas pela hanseníase de acordo com a classificação operacional, além de estabelecer relação com as características socioeconômicas dos pacientes. 


\section{MÉTODOS}

Estudo transversal controlado analítico, realizado em Unidades Básicas de Saúde (UBS) da Estratégia Saúde da Família (ESF) de Teresina-PI, com registro de casos de hanseníase em 2017, correspondendo a 536 pacientes, conforme informações do Sistema de Informação de Agravos de Notificação (SINAN). Realizado no período de novembro de 2018 a março de 2019.

Os critérios de inclusão da amostra foram idade igual ou superior a 18 anos, diagnóstico de hanseníase notificado no SINAN, regularmente cadastrado e acompanhado por equipe da ESF da zona urbana e tratamento poliquimioterápico concluído.

Foram critérios de exclusão: incapacidade de comunicação verbal, deficiência auditiva ou alguma incapacidade cognitiva ou mental que impedisse o preenchimento dos instrumentos de coleta de dados, institucionalizados e não residentes no município.

A amostragem foi probabilística aleatória nas UBS selecionadas. Para a coleta de dados, foi utilizado um formulário contendo variáveis socioeconômicas e a classificação operacional adotada pelo Ministério da Saúde. A escala SALSA foi aplicada para medir a extensão da limitação de atividade e a consciência do risco de se aumentar deficiências durante a realização de determinadas atividades. Esse instrumento é composto por 20 itens, e o escore final de limitação de atividades é obtido pelo somatório dos valores de cada item, podendo variar de 1 a 80 pontos (IKHERA E, et al., 2010).

A consciência de risco foi avaliada por 11 itens, com resultado final podendo variar entre 0 e 11 pontos, sendo que uma consciência crescente dos riscos envolvidos em certas atividades associa-se com escores mais elevados, indicando uma limitação de atividade em razão disso (BRASIL, 2008).

Com objetivo de dimensionar a restrição à participação social de pessoas acometidas pela hanseníase em programas de reabilitação, redução de estigma e integração social foi utilizada a Participation Scale (BARBOSA JC, et al., 2008). O escore final variou de 0 a 90 pontos, a partir do somatório dos valores de cada item. Os graus de restrição foram classificados em: sem restrição significativa ( 0 a 12), restrição leve (13 a 22), moderada (23 a 32), grave (33 a 52) e extrema (53 a 90) (BRASIL, 2008; BRAKEL WH, et al., 2006).

Após assinatura do Termo de Consentimento Livre e Esclarecido (TCLE), foram aplicados o questionário e a escala SALSA e Participation Scale. Os dados foram inseridos em planilha do Microsoft Exce ${ }^{\circledR}$ e processados no softwareIBM® SPSS $\AA^{\circledR}$. Para as variáveis quantitativas foram calculadas médias, desvio padrão, mínimos e máximos; e frequências para as variáveis qualitativas.

$\mathrm{Na}$ análise inferencial das variáveis quantitativas, foi realizado o teste de Kolmogorov-Smirnov para verificação da normalidade dos dados e aplicado o teste $t$ de Student independente. Para as variáveis qualitativas, foram utilizados o teste Qui-quadrado de Pearson ou o teste Exato de Fisher. As análises foram realizadas ao nível de significância de $5 \%$.

O estudo foi realizado em conformidade com as resoluções $n . .0466 / 2012$ e $n^{0}$ 510/2016 do Conselho Nacional de Saúde (CNS) do Ministério da Saúde, bem como as demais resoluções complementares (240/97, 251/97, 292/99 e 340/04), que tratam da pesquisa envolvendo seres humanos, sendo submetido ao Comitê de Ética em Pesquisa (CEP) da Universidade Federal do Piauí (UFPI) e aprovado em 03/12/2018 com o parecer consubstanciado ํㅜㄴ.054.055, assim como pela Comissão de Ética da Fundação Municipal de Saúde de Teresina. 


\section{RESULTADOS E DISCUSSÃO}

Tabela 1 - Características socioeconômicas das pessoas acometidas pela hanseníase conforme classificação operacional, $\mathrm{n}=152$. Teresina, PI, Brasil, 2019.

\begin{tabular}{|c|c|c|c|c|c|}
\hline \multirow{2}{*}{ Variável } & \multicolumn{2}{|c|}{ Multibacilares } & \multicolumn{2}{|c|}{ Paucibacilares } & \multirow{2}{*}{$p$} \\
\hline & M (DP) & n (\%) & $M(D P)$ & n (\%) & \\
\hline Idade (anos) & $\begin{array}{c}49,9 \\
(13,5)\end{array}$ & & $\begin{array}{c}51,1 \\
(13,5)\end{array}$ & & $0,573^{\mathrm{t}}$ \\
\hline Sexo & & & & & $0,001^{9}$ \\
\hline Masculino & & $48(63,2)$ & & $28(36,8)$ & \\
\hline Feminino & & $28(36,8)$ & & $48(63,2)$ & \\
\hline Escolaridade & & & & & $0,026^{9}$ \\
\hline Não alfabetizado & & $12(15,8)$ & & $4(5,3)$ & \\
\hline Fundamental incompleto & & $13(17,1)$ & & $11(14,5)$ & \\
\hline Fundamental completo & & $14(18,4)$ & & $8(10,5)$ & \\
\hline Médio incompleto & & $13(17,1)$ & & $10(13,2)$ & \\
\hline Médio completo & & $16(21,1)$ & & $24(31,6)$ & \\
\hline Superior incompleto & & $6(7,9)$ & & $8(10,5)$ & \\
\hline Superior completo & & $2(2,6)$ & & $11(14,5)$ & \\
\hline Condição atual de emprego & & & & & $0,101^{9}$ \\
\hline Desempregado & & $13(17,1)$ & & $6(7,9)$ & \\
\hline Trabalho em tempo parcial & & $7(9,2)$ & & $14(18,4)$ & \\
\hline Trabalho em tempo integral & & $19(25,0)$ & & $21(27,6)$ & \\
\hline Trabalha por conta própria & & $26(34,2)$ & & $18(23,7)$ & \\
\hline Aposentado & & $11(14,5)$ & & $17(22,4)$ & \\
\hline Situação conjugal & & & & & $0,222^{\dagger}$ \\
\hline Com companheiro & & $56(73,3)$ & & $48(63,2)$ & \\
\hline Sem companheiro & & $20(26,3)$ & & $28(36,8)$ & \\
\hline Convívio familiar & & & & & $1,000^{\dagger}$ \\
\hline Sozinho & & $6(7,9)$ & & $5(6,6)$ & \\
\hline Acompanhado & & $70(92,1)$ & & $71(93,4)$ & \\
\hline Renda familiar & & & & & $1,000^{\dagger}$ \\
\hline Menos de 2 SM & & $42(55,3)$ & & $42(55,3)$ & \\
\hline $2 \mathrm{SM}$ ou mais & & $34(44,7)$ & & $34(44,7)$ & \\
\hline Total & & $\begin{array}{c}76 \\
(100,0)\end{array}$ & & $\begin{array}{c}76 \\
(100,0)\end{array}$ & \\
\hline
\end{tabular}

Legenda: M: média; DP: desvio padrão; SM: $\mathrm{R} \$$ 954,00 (cotação: 01/1/2018); $p$ : significância do teste; $t$ : $\mathrm{t}$ de Student; q: Qui-quadrado de Pearson; $f$ : Exato de Fisher

Fonte: Alencar AR, et al., 2020.

Foram verificadas associações estatisticamente significativas entre classificação operacional da hanseníase e sexo $(p=0,001)$ e escolaridade $(p=0,026)$. Frequência maior do sexo masculino foi identificada no grupo dos multibacilares, com $48(63,2 \%)$, comparativamente a $28(36,8 \%)$ no grupo dos paucibacilares (Tabela 1), o que está de acordo com estudo de Nobre ML, et al. (2017), realizado no Brasil, entre 2001 a 2013. As mulheres desenvolvem uma resposta imunológica mais intensa ao $M$. leprae que os homens, o que justifica uma menor gravidade da doença no sexo feminino (BASSO MEM, SILVA RLF, 2017).

O número de pessoas acometidas pela hanseníase não alfabetizadas também foi maior dentre os multibacilares $12(15,8 \%)$ vs. $4(5,3 \%)$, enquanto o nível superior completo foi muito mais frequente nos paucibacilares $11(14,5 \%)$ vs. $2(2,6 \%)$ (Tabela 1), o que pode ser explicado pelo baixo conhecimento a respeito da patologia e suas manifestações clínicas, contribuindo para a demora na procura pelo serviço de saúde e um possível diagnóstico tardio. Além disso, o desprovimento de conhecimento sobre prevenção e promoção de saúde pode propiciar uma menor adesão ao tratamento medicamentoso e maior índice de incapacidades físicas (MIRANZI SSC, et al., 2010). 
Com relação à idade dos participantes, não houve diferença estatisticamente significativa entre a média ( \pm desvio padrão) de idade dos multibacilares $49,9( \pm 13,5)$ e dos paucibacilares $51,1( \pm 13,5)(p=0,573)($ Tabela 1). A média de idade de multibacilares e paucibacilares situou-se na faixa etária entre a quinta e sexta décadas de vida, já que a hanseníase é uma doença que costuma ser lenta e insidiosa (BRITTON WJ, 2017), o que pode explicar a média de idade mais avançada nos dois grupos encontrada neste estudo.

Dados semelhantes foram encontrados em pesquisa realizada por Sousa GC (2017), com 276 participantes, em unidades básicas de saúde no Maranhão, em que se evidenciou uma associação estatisticamente significativa das variáveis sexo e escolaridade com a classificação operacional, com maior frequência de casos multibacilares entre os homens $73,8 \%(p<0,001)$ e nos indivíduos analfabetos ou com até quatro anos de estudo $36,6 \%(p<0,007)$. A média de idade foi de 51,96 anos $(D P=20,33)$, mas também não apresentou diferença estatisticamente significativa entre os grupos $(p=0,238)$. Entre os paucibacilares, a frequência de indivíduos com mais de doze anos de estudo (75\%) também foi maior em relação aos multibacilares.

Embora não tenha sido observada associação estatisticamente significativa entre classificação operacional e condição atual de emprego $(p=0,101)$, descritivamente, verificou-se maior frequência de desemprego no grupo dos multibacilares $13(17,1 \%)$ vs. paucibacilares $6(7,9 \%)$ (Tabela 1). Isso pode estar associado as formas clínicas multibacilares estarem mais relacionadas com comprometimento neural importante e desenvolvimento de sequelas, ocasionando variados graus de incapacidade e limitação de atividade, além de eventuais deformidades físicas visíveis, associadas ao preconceito e ao estigma que ainda cercam a doença e podem trazer prejuízo no momento de buscar trabalho (SERRA MAAO, et al., 2019).

Não houve associação estatisticamente significativa com situação conjugal ( $p=0,222)$, assim como em relação ao convívio familiar $(p=1,000)$, porém, os participantes relataram viver com companheiro e acompanhados de algum familiar com maior frequência, em ambos os grupos (Tabela 1). Resultados similares foram encontrados em pesquisa realizada por Barboza NA (2017), na cidade de Teresina - PI, com $48,0 \%$ dos participantes vivendo em união estável ou casados. Essa configuração pode representar o retrato do fim do sistema isolacionista que o país viveu, com o continuado processo de desativação dos hospitaiscolônias, e a lenta inserção social dos indivíduos acometidos pela hanseníase ao convívio familiar (CARVALHO KA, 2012).

Também não foi verificada associação estatisticamente significativa com a renda familiar $(p=1,000)$, embora uma menor renda tenha sido observada com maior frequência tanto nos multibacilares $42(55,3 \%)$ quanto nos paucibacilares $42(55,3 \%)$ (Tabela 1). Pesquisa desenvolvida por Ericeira VVL (2017), entre novembro e dezembro de 2016 no município de São Luís - MA, também constatou uma maior frequência de participantes com renda familiar menor que dois salários mínimos $(61,3 \%)$. A baixa renda familiar resulta em condições de moradia mais precárias, suscitando fatores ambientais que facilitam o contágio e a proliferação do bacilo, além de estar relacionada com mais frequência a um estado nutricional mais fragilizado e maior vulnerabilidade de adoecimento (ASSIS IS, et al., 2018).

Tabela 2 - Limitação de atividades da vida diária das pessoas acometidas pela hanseníase conforme classificação operacional, $\mathrm{n}=152$. Teresina, PI, Brasil, 2019.

\begin{tabular}{lccccc}
\hline \multicolumn{1}{c}{ Domínio } & \multicolumn{2}{c}{ Multibacilar } & \multicolumn{2}{c}{ Paucibacilar } & $\boldsymbol{p}$ \\
\hline Visão & M (DP) & Mín-Máx & M (DP) & Mín-Máx & \\
Mobilidade & $1,5(0,6)$ & $1-3$ & $1,2(0,5)$ & $1-3$ & $\mathbf{0 , 0 0 2}$ \\
Autocuidado & $1,8(1,0)$ & $1-4$ & $1,0(0,5)$ & $1-3$ & $<0,001$ \\
Trabalho com as mãos & $1,5(0,7)$ & $1-3$ & $1,0(0,1)$ & $1-1$ & $<0,001$ \\
Destreza das mãos & $1,9(0,8)$ & $1-4$ & $1,1(0,2)$ & $1-2$ & $<\mathbf{0 , 0 0 1}$ \\
\hline Escore global & $1,8(0,7)$ & $1-4$ & $1,2(0,3)$ & $1-2$ & $<\mathbf{0 , 0 0 1}$ \\
\hline
\end{tabular}

Legenda: M: média; DP: desvio padrão; Mín: mínimo; Máx: máximo; $p$ : significância do Teste t de Student. Fonte: Alencar AR, et al., 2020. 
Foi observada diferença estatisticamente significativa entre a média ( \pm desvio padrão) do escore global de limitação de atividade dos multibacilares $35,2( \pm 14,1)$ vs. paucibacilares $21,9( \pm 5,2)(p<0,001)$. Diferenças estatisticamente significativa também foram verificadas em todos os domínios, cujos maiores valores foram dos multibacilares em relação aos paucibacilares: visão 1,5 vs. $1,2(\mathrm{p}=0,002)$; mobilidade dos pés 1,8 vs. 1,0 ( $p<0,001$ ); autocuidado 1,5 vs. 1,0 ( $p<0,001$; $t$ de Student); trabalho com as mãos 1,9 vs. 1,1 ( $<<0,001)$; e destreza das mãos 1,8 vs. 1,2 ( $p<0,001)$. Por meio da escala SALSA, o escore de limitação de atividade para os multibacilares atingiu $90,0 \%$ do escore máximo, resultado próximo ao obtido nos estudos de Rafael AC (2010) e Monteiro LD, et al. (2014) com pontuação atingindo $96,2 \%$ e $82,5 \%$ do total respectivamente. Enquanto que para os paucibacilares o escore máximo correspondeu a $52,5 \%$ do máximo possível, representando uma diferença de 37,5\% para os indivíduos desse grupo (Tabela 2).

Esses dados reforçam a maior gravidade da doença e de sua potencialidade em produzir incapacidades físicas nos indivíduos acometidos pelas formas multibacilares, corroborando com estudo de Sarkar J (2012), realizado na Índia, comparando o desenvolvimento de incapacidades entre indivíduos dos dois grupos da classificação operacional. O maior comprometimento das extremidades é evidenciado na pesquisa de Sousa GC (2017), em que $21,9 \%$ dos pacientes com algum grau de incapacidade apresentavam diminuição ou perda de sensibilidade das mãos ou deformidades, enquanto que $28,2 \%$ apresentavam sintomas semelhantes nos pés.

Tabela 3 - Consciência de risco das pessoas acometidas pela hanseníase conforme classificação operacional, $\mathrm{n}=152$. Teresina, PI, Brasil, 2019.

\begin{tabular}{lccccc}
\hline \multirow{2}{*}{ Domínio } & \multicolumn{2}{c}{ Multibacilar } & \multicolumn{2}{c}{ Paucibacilar } & \multirow{2}{*}{$\boldsymbol{p}$} \\
\cline { 2 - 5 } & $\mathbf{M}(\mathbf{D P})$ & Mín-Máx & $\mathbf{M}(\mathbf{D P})$ & Mín-Máx & \\
\hline Mobilidade & $0,7(1,0)$ & $0-3$ & $0,1(0,3)$ & $0-2$ & $<0,001$ \\
Autocuidado & $0,7(0,2)$ & $0-1$ & $0,0(0,0)$ & $0-0$ & 0,024 \\
Trabalho com as mãos & $1,4(1,4)$ & $0-5$ & $0,1(0,3)$ & $0-1$ & $<0,001$ \\
Destreza das mãos & $0,4(0,5)$ & $0-1$ & $0,0(0,2)$ & $0-1$ & $<0,001$ \\
\hline Escore global & $\mathbf{2 , 6 ( 2 , 5 )}$ & $\mathbf{0 - 9}$ & $\mathbf{0 , 2}(\mathbf{0 , 7})$ & $\mathbf{0 - 4}$ & $<\mathbf{0 , 0 0 1}$ \\
\hline
\end{tabular}

Legenda: M: média; DP: desvio padrão; Mín: mínimo; Máx: máximo; p: significância do Teste t de Student.

Fonte: Alencar AR, et al., 2020.

Houve diferença estatisticamente significativa entre a média ( \pm desvio padrão) do escore global de consciência de risco dos multibacilares $2,6( \pm 2,5)$ vs. paucibacilares $0,2( \pm 0,7)(p<0,001)$. Os multibacilares apresentaram maiores médias comparativamente aos paucibacilares em todos os domínios: mobilidade dos pés 0,7 vs. $0,1(p<0,001)$; autocuidado 0,7 vs. $0,0(p=0,024)$; trabalho com as mãos 1,4 vs. $0,1(p<0,001)$; e destreza com as mãos 0,4 vs. 0,0 ( $p<0,001)$. As diferenças nos escores variaram de 0,4 a 1,3 pontos e 0 escore global máximo de consciência de risco atingido pelo grupo dos multibacilares foi de 9 de um máximo de 11 pontos, enquanto nos paucibacilares foi de 4 pontos (Tabela 3). O valor máximo atingido no grupo dos multibacilares correspondeu a $81,8 \%$ do máximo possível, enquanto nos paucibacilares o máximo compreendeu a 36,4\%. Dado semelhante foi observado por Aben-Athar CYUP, et al. (2017), cuja pesquisa evidenciou uma pontuação máxima de 8 pontos no escore de consciência de risco, correspondendo a 72,7\% do total.

O fato de não conseguirem fisicamente realizar uma atividade específica determinou muitas das situações da consciência de risco, especialmente no grupo dos multibacilares. Fato observado também nos estudos de Barbosa JC, et al. (2008). Esses resultados estão em consonância com o que foi verificado anteriormente em relação à limitação de atividade, pois escores mais elevados na escala de consciência de risco também indicam que há uma maior limitação de atividade em razão disso.

Apesar de todos os domínios apresentarem diferença estatisticamente significativa entre os grupos, o trabalho com as mãos apresentou uma diferença de $65 \%$ maior para os indivíduos acometidos pelas formas multibacilares. O comprometimento de fibras sensitivas e motoras afeta diretamente a execução de atividades diárias que compõem esse domínio de trabalho com as mãos proposto na escala, como cozinhar e preparar alimentos quentes, entre outros. Dessa forma, maior comprometimento da sensibilidade térmica, dolorosa e tátil das mãos nos indivíduos acometidos pelas formas multibacilares (LASTORIA JC, ABREU MAMM, 2014). 
Tabela 4 - Participação social das pessoas acometidas pela hanseníase conforme classificação operacional, $\mathrm{n}=152$. Teresina, PI, Brasil, 2019.

\begin{tabular}{|c|c|c|c|c|c|}
\hline \multirow{2}{*}{ Participação } & \multicolumn{2}{|c|}{ Multibacilar } & \multicolumn{2}{|c|}{ Paucibacilar } & \multirow{2}{*}{$p$} \\
\hline & M (DP) & Mín-Máx & M (DP) & Mín-Máx & \\
\hline Oportunidade de encontrar emprego & $2,0(2,2)$ & $0-5$ & $0,1(0,4)$ & $0-3$ & $<0,001$ \\
\hline Trabalho tanto quanto & $0,6(1,3)$ & $0-5$ & $0,1(0,3)$ & $0-2$ & 0,003 \\
\hline Contribuição econômica & $0,9(1,7)$ & $0-5$ & $0,2(0,8)$ & $0-5$ & 0,002 \\
\hline Viagem para fora da cidade & $1,1(1,1)$ & $0-5$ & $0,4(0,5)$ & $0-2$ & $<0,001$ \\
\hline Ajuda a outras pessoas & $0,6(0,9)$ & $0-5$ & $0,2(0,4)$ & $0-1$ & 0,001 \\
\hline Participação de atividades recreativas e sociais & $1,1(1,2)$ & $0-5$ & $0,3(0,5)$ & $0-2$ & $<0,001$ \\
\hline Ativo socialmente & $1,0(1,1)$ & $0-5$ & $0,1(0,3)$ & $0-1$ & $<0,001$ \\
\hline Visita outras pessoas & $0,8(0,9)$ & $0-5$ & $0,1(0,4)$ & $0-2$ & $<0,001$ \\
\hline À vontade com pessoas novas & $0,2(0,7)$ & $0-5$ & $0,0(0,3)$ & $0-2$ & 0,030 \\
\hline Recebe mesmo respeito & $1,1(1,6)$ & $0-5$ & $0,0(0,3)$ & $0-2$ & $<0,001$ \\
\hline Locomoção dentro e fora de casa & $1,2(1,7)$ & $0-5$ & $0,2(0,6)$ & $0-3$ & $<0,001$ \\
\hline Frequenta locais públicos & $1,1(1,7)$ & $0-5$ & $0,1(0,5)$ & $0-3$ & $<0,001$ \\
\hline Oportunidade de se cuidar & $0,4(1,0)$ & $0-5$ & $0,0(0,0)$ & $0-0$ & 0,001 \\
\hline Serviços de casa & $1,4(1,5)$ & $0-5$ & $0,4(0,8)$ & $0-5$ & $<0,001$ \\
\hline Opinião importante nas discussões familiares & $0,2(0,6)$ & $0-3$ & $0,1(0,3)$ & $0-2$ & 0,149 \\
\hline Come junto com outras pessoas & $0,0(0,2)$ & $0-1$ & $0,0(0,1)$ & $0-1$ & 0,563 \\
\hline Participação de festas e rituais religiosos & $0,8(1,0)$ & $0-5$ & $0,1(0,3)$ & $0-1$ & $<0,001$ \\
\hline Confiança em aprender coisas novas & $0,4(1,0)$ & $0-5$ & $0,1(0,4)$ & $0-2$ & 0,021 \\
\hline Escore global & $14,6(13,5)$ & $0-73$ & $2,6(3,4)$ & $0-14$ & $<0,001$ \\
\hline
\end{tabular}

Legenda: M: média; DP: desvio padrão; Mín: mínimo; Máx: máximo; p: significância do Teste t de Student.

Fonte: Alencar AR, et al., 2020.

Diferença estatisticamente significativa foi verificada entre a média ( \pm desvio padrão) do escore global de participação social dos multibacilares $14,6( \pm 13,5)$ e a dos paucibacilares $2,6( \pm 3,4)(p<0,001)$. O escore máximo de participação atingido pelo grupo dos multibacilares foi de 73 de um máximo de 90 pontos, enquanto nos paucibacilares o escore máximo atingido foi de 14 pontos (Tabela 4).

Os resultados indicaram que a média do escore global de participação para os multibacilares foi de 16,2\% do máximo total, enquanto que para os paucibacilares atingiu apenas $2,9 \%$. O valor máximo de participação social correspondeu a $81,1 \%$ do total que poderia ser alcançado, enquanto nos paucibacilares o máximo atingido representou $15,5 \%$, com diferença de $65,6 \%$.

Em apenas duas categorias de participação social, não houve diferença estatisticamente significativa entre os grupos "opinião importante nas discussões familiares" e "come junto com outras pessoas" ( $p>0,05)$. Nos demais itens, foram observadas médias maiores no grupo dos multibacilares $(p=0,030$ a $p<0,001)$. As diferenças nas médias dos escores dos itens variaram de forma significativa de 0,2 a 1,9 pontos, de modo que a maior diferença foi observada quanto à "oportunidade de encontrar emprego" $(1,9)$, seguida por "receber mesmo respeito" $(1,1)$ (Tabela 4).

A maior diferença na categoria oportunidade de encontrar emprego pode ter relação com uma maior incapacidade física dos indivíduos acometidos pelas formas multibacilares, levando a maior restrição de habilidades que dificultam na hora de conseguir um trabalho. Resultados semelhantes foram encontrados no estudo de Rafael AC (2010), onde os itens da escala de participação referentes ao trabalho foram considerados um grande problema; e no trabalho de Asampong E, et al. (2018), realizado em Gana, que evidenciou um sentimento de rejeição por parte dos empregadores com as pessoas acometidas pela hanseníase, levando a dificuldades na hora de conseguir um trabalho e a inseguranças financeiras.

Quando questionados se recebem o respeito na comunidade tanto quanto seus pares, a diferença entre as médias dos dois grupos também foi bem evidente, reforçando a questão do preconceito firmado na sociedade. Em estudo de Pinheiro MGC e Simpson CA (2017), realizado no Rio Grande do Norte, com familiares de pacientes de um hospital-colônia, as mudanças nas relações sociais mediadas pelo preconceito e estigma puderam ser percebidas em vários relatos dos participantes. 
Gráfico 1 - Classificação de participação social das pessoas acometidas pela hanseníase conforme classificação operacional, n=152. Teresina, PI, Brasil, 2019.

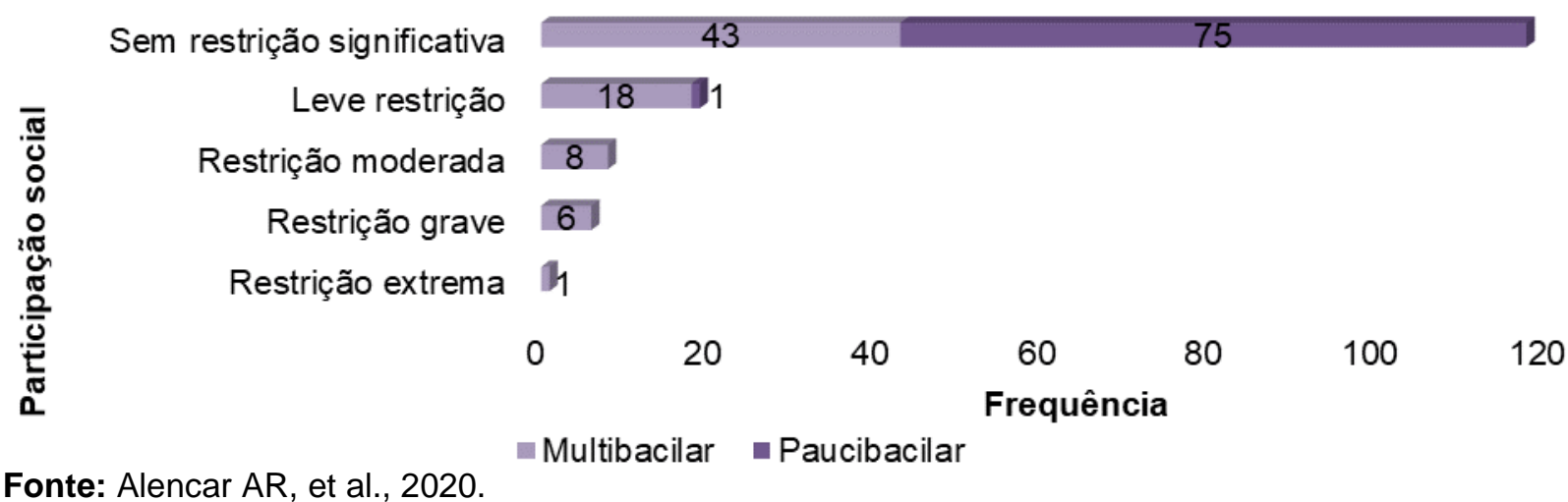

Verificou-se associação estatisticamente significativa entre a participação social e a classificação operacional, de modo que as pessoas acometidas por hanseníase multibacilar apresentaram maior frequência de presença de alguma restrição $33(43,4 \%)$ comparativamente às com hanseníase paucibacilar $1(1,3 \%)$ $(p<0,001$; Fisher). Vale ressaltar que apenas os participantes do grupo dos multibacilares apresentaram restrição moderada 8 (10,5\%), grave $(7,9 \%)$ ou extrema 1 (1,3\%) (gráfico 1).

Embora não seja possível associar a participação social dos indivíduos apenas à hanseníase, a diferença significativa entre os dois grupos operacionais aponta para uma grande influência das formas multibacilares na restrição de participação social das pessoas acometidas, especialmente pelo fato da escala utilizada questionar os indivíduos quanto à sua participação relacionada à hanseníase, comparando-se com alguém semelhante a ele em todos os aspectos, exceto pela doença ou deficiência causada por ela. Isso traz uma maior relevância aos dados apresentados pelo presente estudo.

É importante destacar ainda que toda a amostra é composta por indivíduos que finalizaram o tratamento, sendo esperado, portanto, que a alta por cura favoreça a reinclusão do indivíduo, de forma global; porém o que se verifica no presente estudo é a presença de algum grau de restrição à participação social maior para o grupo multibacilar, de modo que, entre os paucibacilares, apenas um indivíduo apresentou restrição leve à participação, enquanto todos os demais do mesmo grupo não tiveram restrição significativa.

Tendo em vista tudo que foi exposto, é possível entender a gravidade da hanseníase e avaliar a dimensão do dano que ela provoca na vida dos indivíduos acometidos. Sua capacidade em produzir comprometimento neural e gerar incapacidades físicas, promove limitação das atividades diárias e desenvolve nos indivíduos acometidos pela doença uma consciência do risco envolvido em agravar o dano ao realizar determinadas tarefas. Além disso, há uma sequela psicossocial envolvida, reforçada pelo estigma histórico da doença que interfere diretamente na participação social e nas relações interpessoais.

\section{CONCLUSÃO}

O estudo revelou predomínio significativo do sexo masculino e baixa escolaridade com as formas multibacilares, porém não evidenciou diferença importante de faixa etária ou associação da condição atual de emprego, situação conjugal, convívio familiar e renda com a classificação operacional. A diferença dos níveis de limitação de atividade foi expressiva no grupo multibacilar em comparação ao paucibacilar em todos os domínios avaliados. Quanto à consciência do risco envolvido em aumentar as deficiências ao realizar certas atividades diárias, foi constatado que os indivíduos do grupo multibacilar também apresentaram escores maiores em todos os domínios. Tendo em vista que, neste estudo, os participantes do grupo multibacilar apresentaram maiores escores para limitação de atividade, era esperado que também manifestassem maior grau de consciência de risco em aumentar suas deficiências ao realizar determinadas tarefas; pois essas duas variáveis mantêm relação direta. Embora seja difícil mensurar a participação social a partir de uma escala e relacioná-la apenas à hanseníase, a diferença significativa encontrada no estudo entre os dois grupos operacionais indica uma grande influência do acometimento multibacilar na restrição de participação 
social, evidenciando como a hanseníase afeta socialmente a vida das pessoas acometidas, especialmente pela presença de sequelas e pela permanência do estigma. Os domínios relacionados com oportunidade de encontrar emprego e respeito recebido pela comunidade apresentaram as maiores diferenças. Isso revela que o caráter estigmatizante da doença ainda persiste, especialmente no grupo mais acometido por limitações e incapacidades, e reforça a necessidade da adoção de ações de combate ao preconceito e à discriminação.

\section{REFERÊNCIAS}

1. ABEN-ATHAR CYUP, et al. Assessment of the sensory and physical limitations imposed by leprosy in a Brazilian Amazon Population. Rev. Soc. Bras. Med. Trop., vol.50, n.2, pp.223-228. 2017.

2. ASAMPONG E, et al. Caregiver's views on stigmatization and discrimination of people aafected by leprosy in Ghana. PLos. Negl. Trop. Dis., San Francisco, v.12, n.1. 2018.

3. ASSIS IS, et al. Social determinants, their relationship with leprosy risk and temporal trends in a tri-border region in Latin America. PLosNegl. Trop. Dis., San Francisco, v. 12, n.4. 2018.

4. BARBOSA JC, et al. Pós-alta em hanseníase no Ceará: limitação de atividade funcional, consciência do risco e participação social. Rev. Bras. Enferm., v.61, pp.727-733. 2008.

5. BARBOZA NA. Avaliação da atenção primária à saúde no controle da Hanseníase: percepção dos usuários e profissionais de saúde em uma capital brasileira. Programa de Pós-Graduação em Ciências da Saúde, Universidade Federal do Piauí, Teresina. [dissertação de mestrado]. 2017.

6. BASSO MEM, SILVA RLF. Clinical and epidemiological profile of patients affected by Leprosy seen in a reference unit. Rev. Soc. Bras. Clin. Med., v.15, n.1, pp.27-32, jan/mar. 2017.

7. BRAKEL WH, et al. The Participation Scale: measuring a key concept in public health. Disabil. Rehabil., v. 28, n. 4, pp. 193-203. 2006.

8. BRASIL, Ministério da Saúde. Secretaria de Vigilância em Saúde. Departamento de Vigilância Epidemiológica. Manual de prevenção de incapacidades: cadernos de prevenção e reabilitação em hanseníase, Brasília, 1.ed. 2008.

9. BRITTON WJ. Infectious Diseases. 4ed., v. 2, pp. 954-960. 2017.

10. CARVALHO KA. Colônia Santa Izabel: A lepra e o Isolamento em Minas Gerais (1920 - 1960). Univ. Fed. Fluminense, Instituto de Ciências Humanas e Filosofia, Departamento de História [Tese de Doutorado], Niterói. 2012.

11. EICHELMANN K, et al. Leprosy. An update: definition, pathogenesis, classification, diagnosis, and treatment. Actas Dermosifiliogr., Madrid, v. 104, n. 7, pp. 554-63. 2013.

12. ERICEIRA VVL. Hanseníase: Avaliação das limitações das atividades, consciência de risco e participação social. Universidade Federal do Maranhão, Programa de Pós-Graduação em Enfermagem, São Luís. [dissertação de mestrado]. 2017.

13. GASCHIGNARD J, et al. Pauci- and Multibacillary Leprosy: Two Distinct, Genetically Neglected Diseases. PLoSNegl. Trop. Dis., San Francisco, v. 10, n. 5, pp. 1-20. 2016.

14. IKEHARA E, et al. Escala Salsa e grau de Incapacidades da Organização Mundial de Saúde: avaliação da limitação de atividades e deficiência na hanseníase. Acta Fisiatr., v. 17. n. 4. pp. 169 - 174. 2010.

15. LASTORIA JC, ABREU MAMM. Leprosy: review of the epidemiological, clinical, and etiopathogenic aspects - Part 1. An. Bras. Dermatol., v.89, n.2, pp.205-218, mar/apr. 2014.

16. MIRANZI SSC, et al. Perfil epidemiológico da henseníase em um município brasileiro, no período de 2000 a 2006. Rev. Soc. Bras. Med. Trop. Uberaba, v. 43, n. 1, pp.62-67. 2010.

17. MONTEIRO LD, et al. Limited activity and social participation after hospital discharge from leprosy treatment in a hyperendemic area in north Brazil. Rev. bras. epidemiol., São Paulo, v. 17, n. 1, pp. 91-104. 2014.

18. NOBRE ML, et al. Multibacillary leprosy by population groups in Brazil: Lessons from an observational study. PLosNegl. Trop. Dis., San Francisco, v. 11, n. 2. 2017.

19. PINHEIRO MGC, SIMPSON CA. Preconceito, estigma e exclusão social: trajetória de familiares influenciada pelo tratamento asilar da hanseníase. Rev. Enferm. UERJ, Rio de Janeiro, v.25:e1332. 2017.

20. RAFAEL AC. Pacientes em tratamento e pós-alta em hanseníase: estudo comparativo entre os graus de incapacidades preconizados pelo Ministério da Saúde correlacionando-os com as escalas SALSA e participação social. Faculdade de Ciências Médicas da UnB [dissertação de mestrado], Brasília. 2010.

21. SANTOS VS, et al. Functional Activity Limitation and Quality of Life of Leprosy Cases in an Endemic Area in Northeastern Brazil. PLoSNegl. Trop. Dis., San Francisco, v. 9, n. 7, pp. 1-8. 2015.

22. SARKAR J. Disability among new leprosy patients, an issue of concern: an institution-based study in an endemic district for leprosy in the state of West Bengal, Índia. Indian J. Dermatol. Venreol. Leprol, v.78, pp.328-334. 2012.

23. SERRA MAAO, et al. Factors Associated with Multibacillary Leprosy in a Priority Region for Disease Control in Northeastern Brazil: A Retrospective Observational Study. Journal of Tropical Medicine. 2019.

24. SILVA AC, et al. Association between the degree of physical impairment from leprosy and dependence in activities of daily living among the elderly in a health unit in the State of Minas Gerais. Rev. Soc. Bras. Med. Trop., Uberaba, v. 47, n. 2, pp. 212-7. 2014.

25. SOUSA GC. Caracterização clínico-epidemiológica de casos de hanseníase com incapacidades físicas. Rev. Rene, Fortaleza, v.18, n.1, pp. 99-105, jan/fev. 2017.

26. ZAMPARONI V. Leprosy: disease, isolation, and segregation in colonial Mozambique. Hist. cienc. saude-Manguinhos, Rio de Janeiro, v. 24, n. 1, pp. 13-39. 2017. 